\title{
Researching Coal and Rock Character Recognition Based on Wavelet Packet Frequency Band Energy
}

\author{
Guanghui Xue*, Xinying Zhao, Ermeng Liu, Weijian Ding, and Baohua Hu
}

School of Mechanical, Electronic and Information Engineering, China University of Mining and Technology (Beijing), Beijing 100083, China

\begin{abstract}
At present top coal caving is done by workers at the fully mechanized top-coal caving face, where the production rate is low and the workers' safety is under threat. In order to reduce the casualties, realize "less people" even "no people" and the high-efficient and high-yield of coal mine, the coal and rock character recognition was researched on underground, the method based on the wavelet packet frequency band energy analysis, for coal and rock identification was proposed. Using the self-developed mine portable vibration data recorder to pick up the vibration signals at the hydraulic support tail beam under the different conditions of coal caving, gangue caving and roof rock caving in the fully mechanized top-coal caving face. The conclusion has been drawn after analyzing the vibration signals by the wavelet packet frequency band energy: When coal caving, the Edge band focus on $1250 \mathrm{~Hz} \sim 1562.5 \mathrm{~Hz}$ and $2187.5 \mathrm{~Hz} 2500 \mathrm{~Hz}$, when gangue caving, the Edge band focus on $0 \sim 625 \mathrm{~Hz}$ and $937.5 \sim 1250 \mathrm{~Hz}$, when roof rock caving, the Edge band focus on $1875 \sim 2187.5 \mathrm{~Hz}$ and $3750 \sim 4062.5 \mathrm{~Hz}$. The result shows that different edge bands under the different conditions can be used as the criterion for the coal and rock character recognition in the fully mechanized top-coal caving face. And research results could provide a new idea and the method for the coal and rock character recognition.
\end{abstract}

Keywords: Coal and rock characters recognition, fully mechanized top-coal caving face, vibration signals, wavelet packet frequency band energy.

\section{INTRODUCTION}

The fully mechanized top-coal caving face has been widely applied in thick coal seam mining in China, and is used as one of the main methods of thick coal seam mining $[1,2]$ for high yield and high efficiency, low consumption, safety and good economic benefits. At present, the close timing of coal export during the top coal caving are judged by the worker's intuition and experience based on whether there are gangue and in how much quantity in the coal (coal and rock characters). Due to harsh environment, dust, and darkness, it is difficult for workers to judge the coal and rock characters that inevitably cause their "less fall" and "over fall" status. "Less fall" not only leads to the low coal recovery rate, but also increases the risk of goaf spontaneous combustion. "Over fall" would result in coal quality decline, invalid transport, increased washed coal cost and render coal mine workers' health and personal safety under serious threat.

This study involves: using the self-developed $\mathrm{YHJ}(\mathrm{C})$ mine portable vibration recorder to pick-up the vibration response signal at the hydraulic support tail beam excited by coal, gangue and roof rock caving; using the method of the wavelet packet frequency band energy to analyze those data; and finding the difference under those conditions.

*Address correspondence to this author at School of Mechanical, Electronic and Information Engineering, China University of Mining and Technology (Beijing), Beijing 100083, China;

Tel: +8613581534915; E-mail: xgh@cumtb.edu.cn

\section{WAVELET PACKET FREQUENCY BAND EN- ERGY}

\subsection{Wavelet Decomposition}

Wavelet decomposition $[3,4]$ is a time-frequency localization analyzing method to realize time and frequency change at the same time.

Satisfied allow conditions:

$\int_{-\infty}^{+\infty} \psi(t) d t=0$

$\psi(t)$ is mother wavelet function. By flexing and translating $\psi(t)$, get

$\psi_{a, b}(t)=|a|^{-\frac{1}{2}} \psi\left(\frac{t-b}{a}\right)$

$a$ reflects the scale function, $b$ shows translational position at $t$ axis, $\psi_{a, b}(t)$ is the wavelet function.

In practical application, signal and parameter $a, b$ in wavelet function are discrete variable. Setting $a=2^{m}$, $b=2^{m} n$, we can get

$\psi_{m, n}(t)=2^{-\frac{m}{2}} \psi\left(2^{-m} t-n\right),(m, n \in Z)$

$\psi_{m, n}(t)$ is wavelet function generated binary discrete wavelet. 


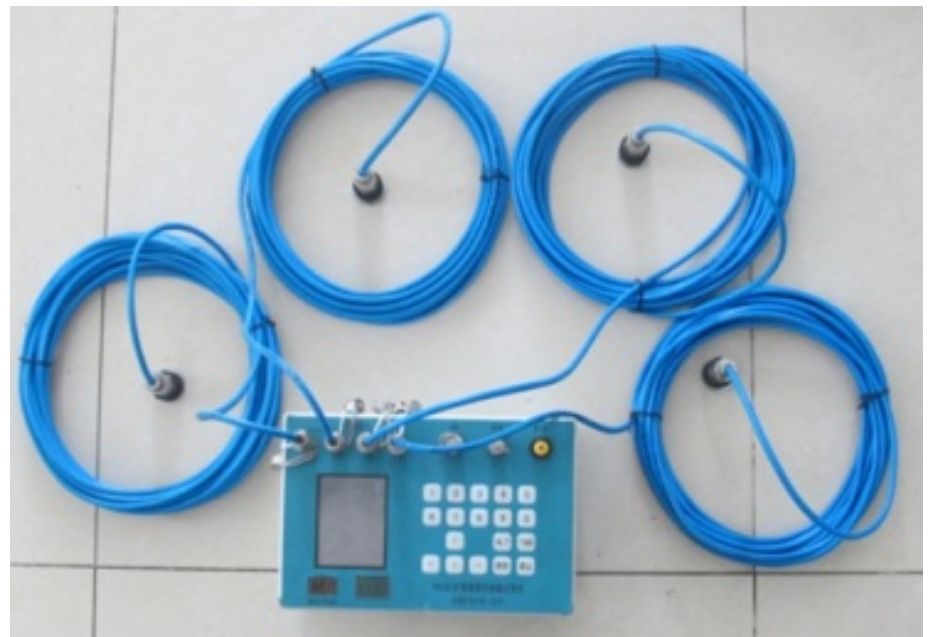

Fig. (1). The $\mathrm{YHJ}(\mathrm{C})$ mine portable vibration recorder.

If binary discrete wavelet $\psi_{m, n}(t)$ satisfies the condition of orthonormal basis of normed linear space $L^{2}(R)$, and the binary orthogonal scaling function is generated by mother wavelet function $\psi(t)$, get

$\varphi_{m, n}(t)=2^{-\frac{m}{2}} \varphi\left(2^{-m} t-n\right)(m, n \in Z)$

$\psi_{m, n}(t)$ 's wavelet space $W_{m}$ and $\varphi_{m, n}(t)$ 's scale space $V_{m}$ are complementation in the $L^{2}(R)$ 's space. Scale space $V_{m-1}$ is the straight sum of $V_{m}$ and $W_{m}$.

So $x(t)$ 's orthogonal wavelet decomposition is

$x(t)=\sum_{m=1}^{N} \sum_{n \in Z} d_{n}^{m} \psi_{m, n}(t)+\sum_{n \in Z} c_{n}^{N} \varphi_{N, n}(t)$

$N$ is the exploded layer number, $d_{n}^{m}$ is wavelet coefficients, $c_{n}^{N}$ is scale coefficients at the $N$ layer. $\varphi_{N, n}(t)$ is binary orthogonal scaling function when $m=N$.

\subsection{Wavelet Packet Decomposition}

Wavelet decomposition only decomposes the low frequency part and gives up decomposing of the high frequency part. But wavelet packet decomposition [5] make up for the shortage.

Assuming that $\varphi(x)$ and $\psi(x)$ are one of $V_{0}$ and $W_{0}$ orthonormal basis respectively. Because $V_{0} \in V_{-1}$ and $W_{0} \in V_{-1}, \varphi(x)$ and $\psi(x)$ belong to the space of $V_{-1}$, namely $\varphi(x), \psi(x)$ are linear unfolded by orthogonal basis $\varphi_{-1, n}(x)$ in the space of $V_{-1}$ :

$$
\begin{aligned}
& \varphi(x)=\sqrt{2} \sum_{k} h_{0}(k) \varphi(2 x-k) \\
& \psi(x)=\sqrt{2} \sum_{k} g(k) \varphi(2 x-k)
\end{aligned}
$$

Among them, $h_{0}(k)=\left\langle\varphi, \varphi_{-1, k}\right\rangle, g(k)=\left\langle\psi, \varphi_{-1, k}\right\rangle$.

Assuming $u_{0}(x)=\varphi(x), u_{1}(x)=\psi(x)$, two dimension equation can be written:

$$
\begin{aligned}
& u_{2 n}(x)=\sqrt{2} \sum_{k} h(k) u_{n}(2 x-k) \\
& u_{2 n-1}(x)=\sqrt{2} \sum_{k} g(k) u_{n}(2 x-k)
\end{aligned}
$$

$u_{n}$ is orthogonal wavelet packet of packet orthogonal scaling function $\varphi(x)$.

\subsection{Frequency Band Energy Analysis.}

Sum of the energy of each band signal:

$E_{j}=\sum_{k=1}^{n} X_{k}^{2}(t)$

$E_{j}$ represents the wavelet packet energy at last $\mathrm{j}$ layer, $X_{k}$ represents spectrum coefficient of discrete points after being decomposed by layer wavelet packet.

Use the total energy $E_{0}=\sqrt{\sum_{i=1}^{n} E_{j}^{2}}$ to normalize the energy signal feature vector $E=\left[E_{1}, E_{2}, E_{3}, E_{4} \cdots E_{n}\right]$, get

$$
E^{\prime}=\left[E_{1} / E_{0}, E_{2} / E_{0}, E_{3} / E_{0}, E_{4} / E_{0} \cdots E_{n} / E_{0}\right]
$$

The above make the theoretical foundation of frequency band energy of wavelet packet analysis.

\section{ACQUIRE THE COAL AND ROCK'S VIBRATION SIGNAL}

\subsection{Mine Portable Vibration Recorder and Test}

$\mathrm{YHJ}(\mathrm{C})$ mine portable vibration recorder [6,7], as shown in Fig. (1), is the self-developed 4 channel coal mine vibra- 


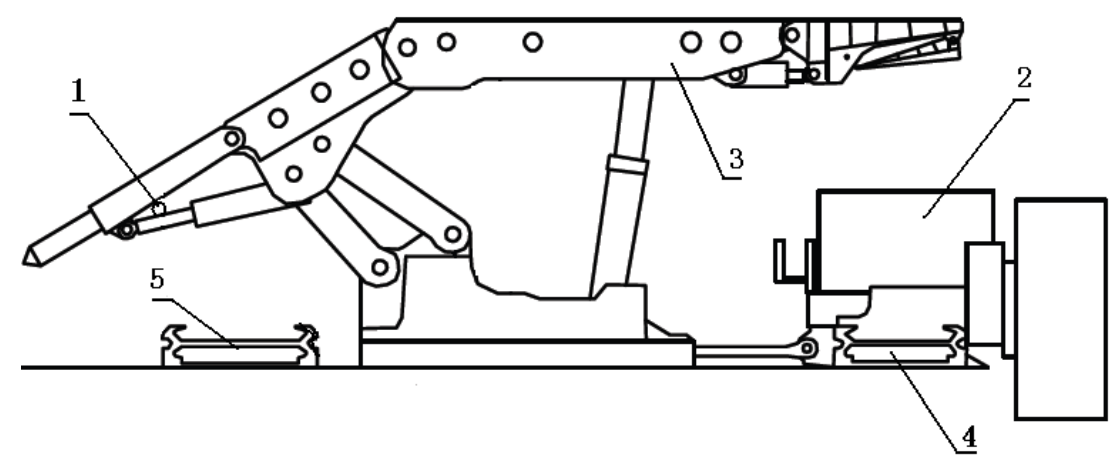

1-point at back beam; 2- coal winning machine; 3- hydraulic support; 4- front scraper conveyor; 5-Rear scraper conveyor.

Fig. (2). Site equipment layout and measuring point location.

tion signal acquisition instrument. Its sampling frequency is $0 \sim 250 \mathrm{kHz}$, and can collect, record and store the mine equipment vibration signal data, and provide technical support and guidance for the daily maintenance and repair of the equipment.

Site equipment layout and observation points. This test was carried out at 922 fully mechanized top-coal caving face of Dongpo coal mine in Shanxi province. The vibration data was collected at the hydraulic support tail beam under the coal caving, gangue caving and roof rock caving conditions. Sampling frequency was $10 \mathrm{kHz}$. The point at $143 \#$ hydraulic support was selected after field investigation and communication with workers. Fig. (2) shows arrangement of measurement points, and $1 \#$ point collected the vibration data at the hydraulic support beam normal direction.

Fig. (3) is the time domain waveform figure under various work conditions.

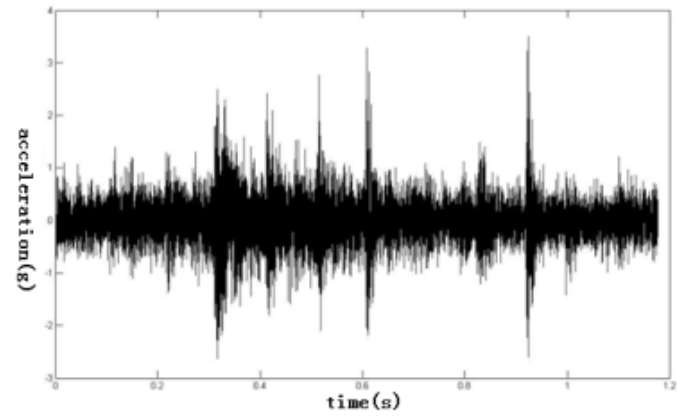

(a) coal caving

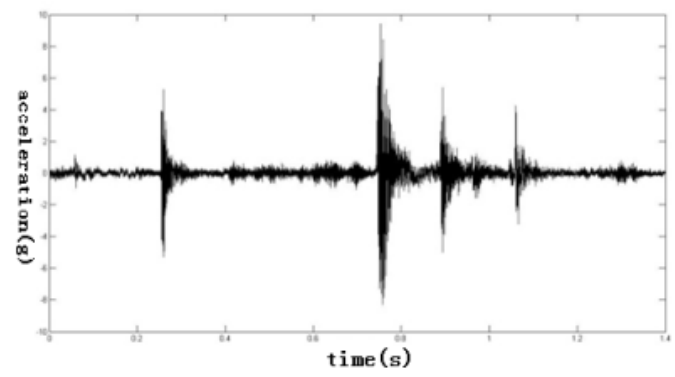

(b) gangue caving

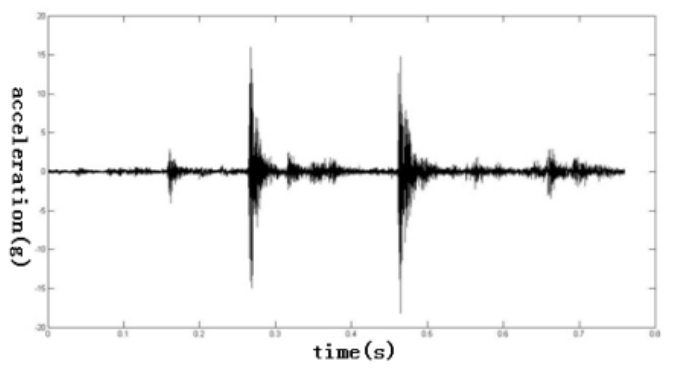

(c) roof rock caving

Fig. (3). The time history of vibration signal at the measurement point of the hydraulic support tail beam under various work conditions.

Results can be obtained from Fig. (3).

The vibration amplitude is about $3 \mathrm{~g}$ generated under the condition of coal caving. No obvious phenomenon of impact load happened.

The vibration amplitude is about $8 \mathrm{~g}$ generated under the condition of gangue caving. Obvious phenomenon of impact load happened.

The vibration amplitude is about $15 \mathrm{~g}$ generated under the condition of roof rock caving. Obvious phenomenon of impact load happened.

\subsection{Frequency Band Energy of Wavelet Packet Analysis.}

Based on the regularity comparability of wavelet coefficient, choose the $\mathrm{db} 2$ wavelet packet basis, and 4 layer of wavelet packet to analyse the vibration signals. $0 \sim 5000 \mathrm{~Hz}$ have been decomposed to 16 bands, and getting the time frequency diagram, as shown in Fig. (4).

Calculate the energy of each band under various work conditions, and the energy histograms have been gotten as normalized, as shown in Fig. (5).

From Fig. (5) conclusions can be drawn:

When coal caving, the energy between A5 band and A8 band, namely $1250 \mathrm{~Hz} 1562.5 \mathrm{~Hz}$ and $2187.5 \mathrm{~Hz} \sim 2500 \mathrm{~Hz}$ is higher than in other conditions. 

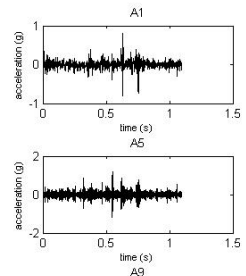

time (3)
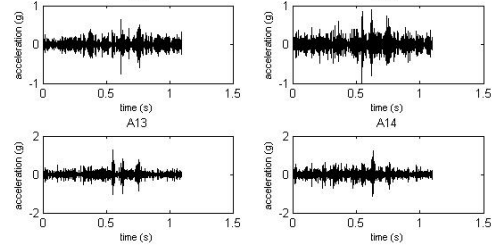

(mes)(s)

(a) coal caving
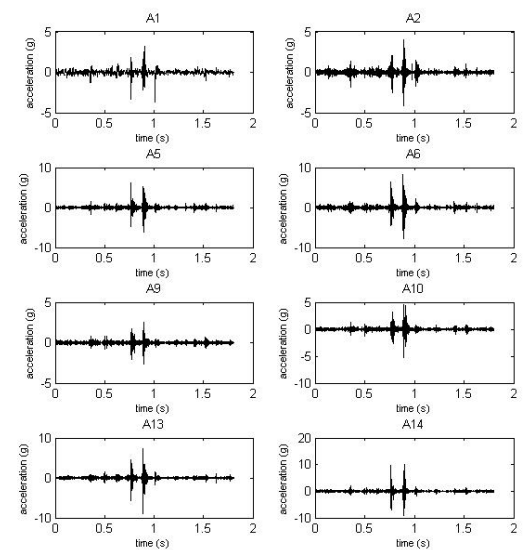

time (s)

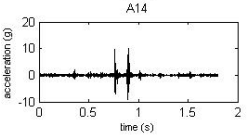

(b) gangue caving
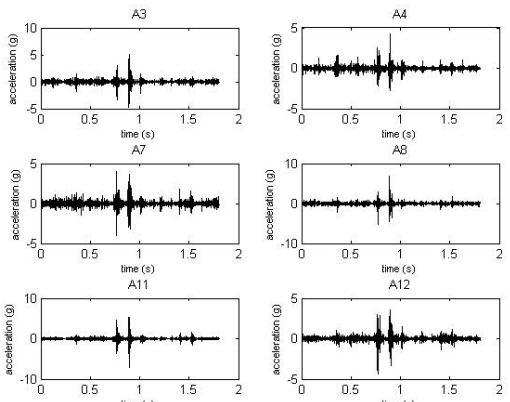

time $(s)$
A15
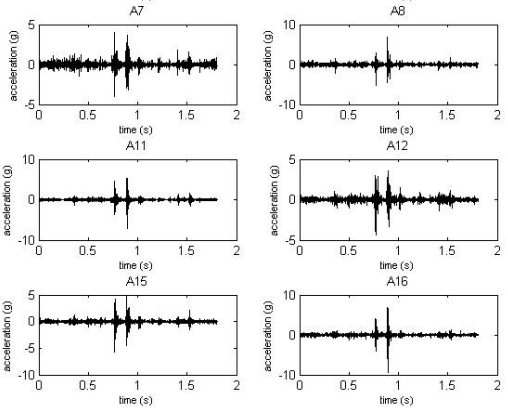
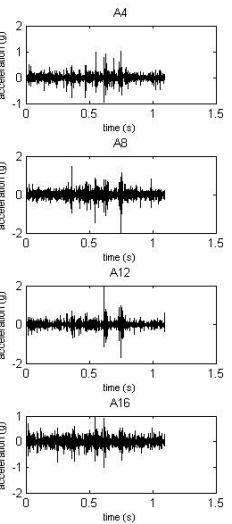
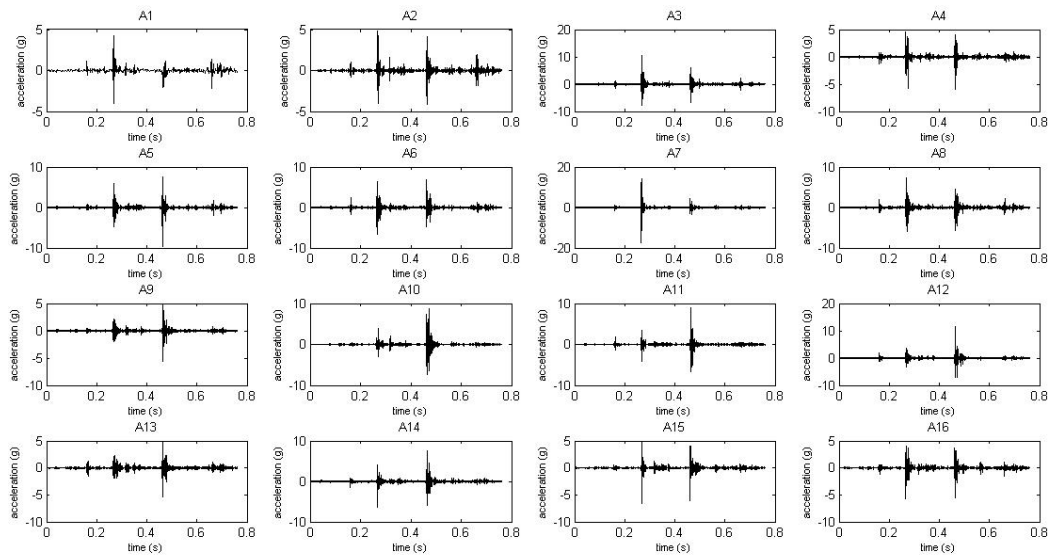

(c) roof rock caving

Fig. (4). Wavelet packet decomposition of vibration signals under various work conditions.

When gangue caving, the energy among A1 band, A2 band and A4 band, namely $0 \mathrm{~Hz} \sim 625 \mathrm{~Hz}$ and $37.5 \mathrm{~Hz} \sim 1250$ $\mathrm{Hz}$ is higher than in other conditions.

When roof rock caving, the energy between $\mathrm{A} 7$ band and A13 band, namely $1875 \mathrm{~Hz} \sim 2187.5 \mathrm{~Hz}$ and $3750 \mathrm{~Hz}$ $\sim 4062.5 \mathrm{~Hz}$ is higher than in other conditions.

\section{DISCUSSING THE DATA ANALYSIS RE- SULTS}

The results found in this paper are universal for different coal mines and different working faces. But the specific frequency band threshold under various conditions applies only to this test of working face. For other fully-mechanized cav- 


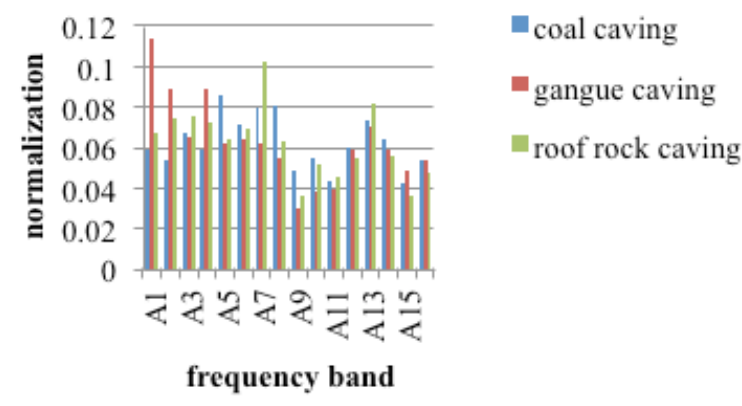

Fig. (5). The frequency band normalized energy histograms of vibration signal at the measurement point of the hydraulic support tail beam under various work conditions.

ing face, the method proposed in this paper along with test and analysis can be used to ensure the threshold under the various conditions. Concrete judgment of coal and rock character recognition can been done.

\section{SUMMARY}

The amplitude at the time domain waveform figure is different at the measurement point of the hydraulic support tail beam under various work conditions. The amplitude is about $3 \mathrm{~g}$ when coal caving, $9 \mathrm{~g}$ when gangue caving and $15 \mathrm{~g}$ when roof rock caving.

The wavelet packet frequency band energy analysis has shown the following: the edge band concentrate on 1250 $\mathrm{Hz} \sim 1562.5 \mathrm{~Hz}$ and $2187.5 \mathrm{~Hz} \sim 2500 \mathrm{~Hz}$ when coal caving, 0 $\mathrm{Hz} \sim 625 \mathrm{~Hz}$ 与 $937.5 \mathrm{~Hz} \sim 1250 \mathrm{~Hz}$ when gangue caving, $1875 \mathrm{~Hz} 2187.5 \mathrm{~Hz}$ and $3750 \mathrm{~Hz} \sim 4062.5 \mathrm{~Hz}$ when roof rock caving. The edge band is different obviously, and it can be the gist of coal and rock character recognition under various working conditions.

The method of the wavelet packet frequency band energy analysis can decompose the coal and rock's vibration signal of nonlinear and non-stationary into bands, calculate the energy of each band to find the difference. It can be the powerful tool of coal and rock recognition at fully mechanized topcoal caving face.

\section{CONFLICT OF INTEREST}

The authors confirm that this article content has no conflict of interest.

\section{ACKNOWLEDGEMENTS}

This work was supported by the National Key Basic Research and Development Project (No.2014CB046300), and Fundamental Research Funds for the Central Universities (No.2009QJ16).

\section{REFERENCES}

[1] J. M. Li, and Z. Y. Zhang, "The fully mechanized top coal caving mining technology in complicated seam of Kailuan mining area", Journal of China Coal Society, vol. 11, pp. 1794-1799, 2010.

[2] Z. J. Wang, and Z. W. Zhang, "Mining technology of $300 \mathrm{~m}$ long fully-mechanized top coal caving mining face with annual production of over 10 millions tons", Journal of China Coal Society, vol. 3, pp. 32-35, 2014.

[3] L. Zhou, X. Xia, Y. J. Wan, H. Zhang, and P. Lei, "Harmonic detection based on wavelet transform", Transactions of China Electrotechnical Society, vol. 9, pp. 68-73, 2006.

[4] Z.L. Liu, "Evaluation on developing level of unban agglomeration derived from resources exploration", Journal of Applied Sciences, vol. 13, no. 21, pp. 4702-4707, 2013.

[5] F. T. Wang, X. J. Ma, and Y. Zou, "Local power feature extraction method of frequency bands based on wavelet packet decomposition", Transactions of the Chinese Society for Agricultural Machinery, vol. 5, pp. 177-179. 2004.

[6] J. J. Yang, G. H. Xue, G. R. Zhao, S. Y. Wu, and M. Wu, "Research and development on mine intrinsic safe mode vibration sensor", Coal Science and Technology, vol. 2, pp. 71-74, 2013.

[7] G. H. Xue, M. Wu, and B. Zhou, "Development of mine portable digital recorder", Coal Science and Technology, vol. 5, pp. 52-54, 2004 .

Received: September 16, 2014

(C) Xue et al.; Licensee Bentham Open.

This is an open access article licensed under the terms of the Creative Commons Attribution Non-Commercial License (http://creativecommons.org/licenses/by-nc/4.0/) which permits unrestricted, non-commercial use, distribution and reproduction in any medium, provided the work is properly cited. 